\title{
Right Paraduodenal Hernia in an Adult Patient: Diagnostic Approach and Surgical Management
}

\author{
Carlos M. Nuño-Guzmána José Arróniz-Jáuregui ${ }^{a}$ \\ Cuauhtémoc Hernández-González ${ }^{\mathrm{a}} \quad$ Francisco Reyes-Macías ${ }^{\mathrm{a}}$ \\ Rosa Nava-Garibaldia Francisco Guerrero-Díaz ${ }^{a}$ \\ José Martínez-Chávez ${ }^{\mathrm{a}} \quad$ Josué Solís-Ugalde ${ }^{\mathrm{b}}$
}

Departments of a General Surgery and ${ }^{b}$ Radiology, Antiguo Hospital Civil de

Guadalajara 'Fray Antonio Alcalde', Guadalajara, Mexico

\section{Key Words}

Paraduodenal hernia $\cdot$ Internal hernia $\cdot$ Intestinal obstruction

\begin{abstract}
Paraduodenal hernia, a rare congenital anomaly which arises from an error of rotation of the midgut, is the most common type of intraabdominal hernia. There are two variants, right and left paraduodenal hernia, the right being less common. We report the case of a 41-year-old patient with a right paraduodenal hernia with a 6-month history of intermittent episodes of intestinal obstruction. Diagnosis was established by CT scan and upper gastrointestinal series with small bowel follow-through. In a planned laparotomy, herniation of the small bowel loops through the fossa of Waldeyer was found. Division of the lateral right attachments of the colon opened the hernia sac widely, replacing the pre- and postarterial segments of the intestine in the positions they would normally occupy at the end of the first stage of rotation during embryonic development. Six months after the surgery, after an uneventful recovery, the patient remains free of symptoms.
\end{abstract}

\section{Introduction}

Internal hernia is defined as a fossa or defect of unusual size within a body cavity, into which intestines may intrude and become incarcerated or strangulated. The origins of internal hernias are multiple and include congenital defects, inflammation, infectious processes, trauma, and postoperative defects [1]. Based on autopsy findings, their incidence has been reported to be between 0.2 and $0.9 \%$. The most common presentation 
of an incarcerated internal hernia is acute small bowel obstruction, whereas $4.1 \%$ of all intestinal obstructions are caused by internal hernias [2].

Paraduodenal hernia is the most common type of internal hernia, accounting for 53\% of reported cases [3]. The term 'paraduodenal hernia' refers to a hernia of the entire small bowel, or part of it, into a sac derived from folds of peritoneum and fossae normally found at the terminal or 4th portion of the duodenum. It represents a rare congenital anomaly which arises from an error of rotation of the midgut. There are two variants, right and left paraduodenal hernia, the right being less common [4].

A right paraduodenal hernia is formed when the prearterial limb fails to rotate around the superior mesenteric artery (SMA). The prearterial segment is the portion cephalic to the vitello-mesenteric duct and comprises the distal duodenum and the entire small bowel to the distal ileum. Therefore, a portion of the small bowel remains to the right of the SMA. Fusion of the ascending colonic mesentery to the retroperitoneum causes entrapment of the bowel within the primitive coelom, affecting from a single loop to the entire small bowel. The result is a hernia orifice that is always to the right of the midline and usually faces medially and slightly downward. The mesentery of the ascending colon and a portion of the transverse colon make up the anterior wall of the sac, while the SMA and ileocolic artery lie in the free edge of the sac $[1,4]$.

Signs and symptoms of paraduodenal hernia are extremely variable and may occur at any age [4]. The majority of cases are noted between the 4th and 6th decades of life. The average age at diagnosis described is 38.5 years [5]. Based on the Japanese literature, $70 \%$ of cases were male, and the mean age at onset was 39.5 years [6]. The most common presentation is acute small bowel obstruction, with crampy abdominal pain, nausea, vomiting and distension. The patient may complain of vague and chronic abdominal pain or periodic distension, which results from partial obstruction. Intermittent postprandial abdominal discomfort or tenderness may result in weight loss. The severity of signs and symptoms is directly proportional to the degree of obstruction $[3-5,7,8]$.

A paraduodenal hernia may be discovered incidentally at autopsy $[4,8]$. In many cases it causes no symptoms and diagnosis may be made when a barium x-ray examination shows the small bowel either to the right or left of the midline in the abdomen [4].

We report a case of right paraduodenal hernia in an adult patient, followed by a review of the literature.

\section{Case Report}

A 41-year-old male patient was referred to our Department of General Surgery with a 6-month history of intermittent episodes of postprandial nausea, vomiting, abdominal pain and distension, which aggravated during the last 3 months, importantly affecting oral intake. There was no history of previous abdominal surgery. Physical examination revealed mild distension and tenderness of the upper abdominal quadrants, which appeared tympanic at percussion. Blood analysis was unremarkable. An abdominal CT scan with oral and intravenous contrast demonstrated an encapsulated cluster of small bowel loops occupying mainly the right upper quadrant, lateral to the duodenum, with the transverse colon located inferiorly (fig. 1). Upper gastrointestinal series with small bowel follow-through showed a large collection of contrast-filled dilated bowel loops in the right upper aspect of the abdomen and absence of small bowel loops in the lower abdomen, confirming the diagnosis of a right paraduodenal hernia (fig. 2). The patient underwent a laparotomy; a large sac containing dilated small bowel loops was identified as demonstrated with radiologic studies ( $\underline{\text { fig. }} 3 \mathrm{~A}$ ). The ascending colon was normally 
located, whereas the transverse colon was displaced inferiorly. When the transverse colon was retracted in cephalic direction, the right paraduodenal hernia, through which most of the small bowel protruded, was identified. The medial aspect of the hernia neck included the superior mesenteric vessels, under a certain degree of tension due to the distended loops passing through, opposing difficulty to their reduction (fig. 3B). Surgical correction was accomplished by dividing the lateral attachment of the ascending colon, opening the hernia sac widely and rotating the right colon medially, connecting the small bowel with the abdominal cavity. There were no other abnormalities at abdominal exploration. After an uneventful recovery, the patient initiated oral intake at the second postoperative day, and 6 months after the surgery the patient remains free of symptoms in a good nutritional status.

\section{Discussion}

The term 'paraduodenal hernia' refers to a hernia of the entire small bowel, or part of it, into a sac derived from folds of peritoneum and fossae normally found at the terminal or 4th portion of the duodenum. No less than 10 such peritoneal fossae have been described, but the most frequently encountered are: inferior paraduodenal fossa of Treitz (60\%), combined superior and inferior paraduodenal fossae (30\%), superior paraduodenal fossa (5\%), paraduodenal fossa of Landzert (2\%), duodenojejunal or mesocolic fossa (2\%), and fossa of Waldeyer $(1 \%)[9,10]$. Approximately $75 \%$ of paraduodenal hernias occur on the left side of the abdomen and involve the paraduodenal fossa of Landzert; 25\% develop on the right, involving the fossa of Waldeyer, located in the mesojejunum, beneath the SMA and immediately below the duodenum. Their presence is more common in men than women, with a ratio of $3: 1[2,7,9]$.

Paraduodenal hernias are derived from an error of rotation of the midgut, the portion of the intestinal tract receiving its blood from the SMA. It is divided into two segments. The portion cephalic to the vitello-mesenteric duct is called the prearterial segment and comprises the distal duodenum and the entire small bowel to the distal ileum. The caudal portion, or postarterial segment, comprises the distal ileum, cecum, appendix, ascending colon and proximal two thirds of the transverse colon [4]. At about the 4th to 5th week of fetal development, the midgut rate growth exceeds the rate growth of the body stalk and elongates, forming the primary intestinal loop. Due to this rapid elongation as well as the increasing size of the liver, a physiologic herniation at the umbilical ring occurs. By about the 10th to 12th week the abdominal cavity has increased in size and the midgut has gradually returned within it. The midgut has now rotated $90^{\circ}$ in a counterclockwise direction on the axis of the SMA. The prearterial segment occupies the right side of the abdominal cavity, and the postarterial segment the left side. Arrest at this point of rotation will produce the relatively common picture of nonrotation of the intestine. Normally the prearterial segment continues to rotate an additional $180^{\circ}$ counterclockwise, first behind and then to the left of the SMA, so that it comes to lie to the left of the midline in the abdominal cavity. The postarterial segment also rotates, led by the cecum, which passes counterclockwise anterior to the SMA into the right upper quadrant. The cecum may not complete its descent to its normal position in the right lower quadrant until about the end of the 5th month of life of the embryo. Later, fusion of the mesenteries and fixation of the midgut occurs, from the ligament of Treitz in the left upper quadrant to the cecum in the right lower quadrant. The ascending and descending colon fuse with the retroperitoneum. As mentioned, a right paraduodenal hernia is formed when the prearterial limb fails to rotate around the SMA, and a portion of the small bowel remains to the right of it $[1,4]$. 
The age of our case falls in the most common range reported $[5,6]$. The 6-month history of intermittent episodes of postprandial nausea, vomiting, abdominal pain and distension was suggestive of a partial small intestinal obstruction. Unfortunately, these nonspecific symptoms are often mistakenly attributed to biliary disease, gastritis, or gastroesophageal reflux. Physical examination is usually not revealing unless the hernia is large enough to produce an abdominal mass or causes intestinal obstruction [5]. Distension of the abdomen may be eccentric, situated on the corresponding side of the hernia. Palpation may confirm a vague soft to firm mass [8]. Our patient manifested with mild distension and tenderness of the upper abdominal quadrants, which appeared tympanic at percussion.

The first correct preoperative diagnosis of a paraduodenal hernia was made by Kummer in 1921, who described its presence by a barium swallow [5]. Later, Taylor in 1930 diagnosed a case of right paraduodenal hernia by radiologic appearances [11]. A plain abdominal film will usually show an agglomeration of dilated small bowel loops. A definitive preoperative diagnosis may still be made by an upper gastrointestinal series with small bowel follow-through that shows an ovoid collection of contrast-filled small bowel loops either to the right or left of the midline of the abdomen. In a right paraduodenal hernia, as seen in our case, collection of bowel loops is noted lateral and inferior to the descending duodenum, in the right half of the transverse mesocolon, or behind the ascending mesocolon. These bowel loops cannot be displaced despite repositioning of the patient. There is absence of small bowel in the pelvis, except when most of the terminal ileum is outside the hernia sac. Barium enema examination may show a colon in its usual position or the cecum may be only incompletely rotated and lie in the right upper quadrant $[4,5,12]$.

CT has been reported to demonstrate both right and left paraduodenal hernias. The classic CT findings of a right paraduodenal hernia were visualized in our patient: clustering of small bowel loops in the right midabdomen. Small bowel obstruction may be present with dilated loops containing air-fluid levels. Vascular findings include jejunal branches of the SMA and superior mesenteric vein looping posteriorly and to the right to supply the herniated loops. Additional findings include the presence of the SMA, ileocolic artery, and right colic vein in the anterior margin of the hernial sac neck, displaced anteriorly $[5,12]$.

The surgical treatment of a right paraduodenal hernia, as performed in the present case, is to replace the pre- and postarterial intestinal segments to their normal positions at the end of the first stage of rotation, with the duodenum, jejunum and most of the ileum to the right, and the terminal ileum, cecum and colon on the left of the midline. This is their relation in the nonrotation of the intestine and will be accomplished by dividing the right lateral attachments of the colon and transferring it to the left side of the abdomen. Therefore, the hernia sac is widely opened and the small opening through which the ileum passes is eliminated. The hernia sac will now be part of the general peritoneal cavity [4]. Since the SMA and its branches to the cecum and ascending colon lie in the anterior wall of the hernia sac, its injury may result if hernia reduction is attempted by opening the sac in this area $[4,5]$. Surgical management has been successfully performed with the laparoscopic approach in patients with no bowel necrosis or severe dilatation [13].

Operative treatment of hernia found incidentally at laparotomy has been debated $[4,5]$. Paraduodenal hernias carry about a 50\% lifetime risk of incarceration, which 
may lead to bowel obstruction and strangulation. Therefore, treatment has been recommended. The mortality rate is not clear but approximates $20-50 \%$, due to the large proportion of patients with intestinal obstruction and ischemia requiring emergency surgery. There is a poorer prognosis if strangulation occurs and a long segment of small bowel is rendered ischemic. Moreover, this may result from delay in intervention, as signs of peritonitis may be masked by the retroperitoneal position of the hernia $[14,15]$.

The case reported herein is a male patient who showed proximal obstructive symptoms, being evaluated by contrast-enhanced gastrointestinal studies under nonurgent circumstances, which allowed a planned laparotomy. As recommended, the surgical correction avoided a reduction attempt of herniated bowel loops and hence the possibility of injury to the superior mesenteric vessels $[4,5]$.

\section{Disclosure Statement}

The authors have no conflicts of interest, sponsorship or funding arrangements to declare.

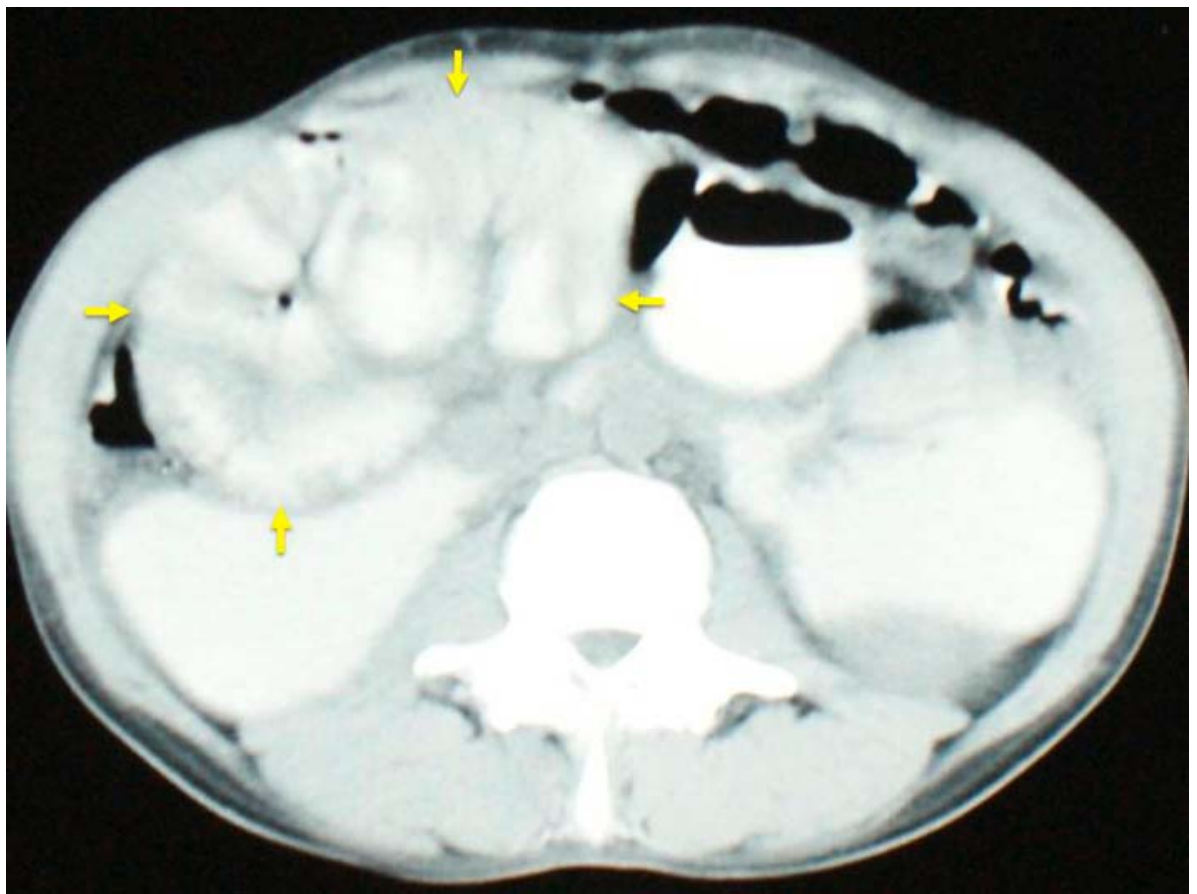

Fig. 1. Computed abdominal tomography image with intravenous and oral contrast. The classic cluster of small bowel loops in the right upper quadrant can be seen (arrows). 


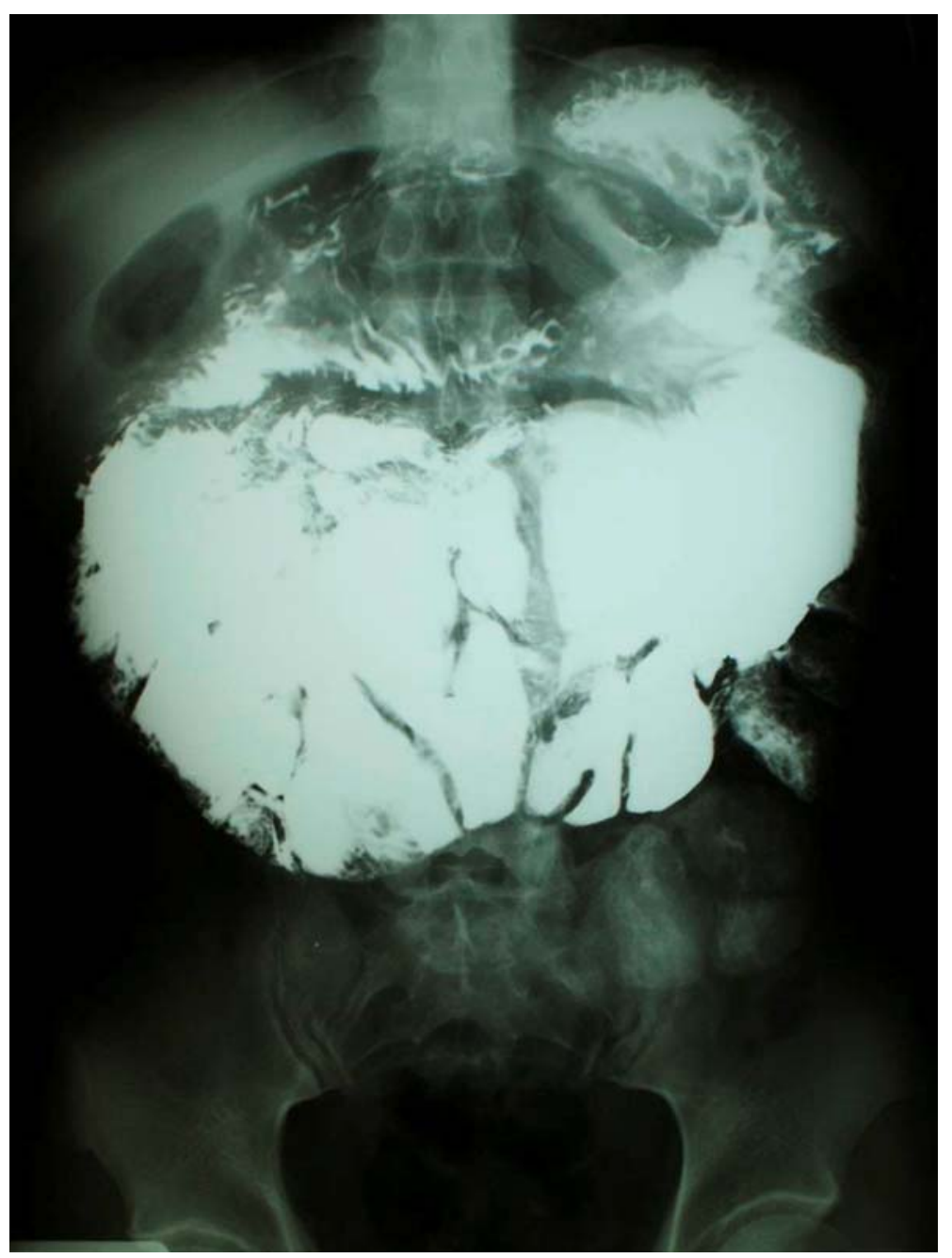

Fig. 2. Small bowel follow-through radiologic image. Contrast-filled dilated small bowel loops in the right upper aspect of the abdomen and absence of small bowel loops in the lower abdomen are depicted. 


\begin{tabular}{|c|c|c|c|}
\hline $\begin{array}{l}\text { Case Reports in } \\
\text { astroenterology }\end{array}$ & $\begin{array}{l}\text { Case Rep Gastroenterol 2011;5:479-486 } \\
\text { DOI: 10.1159/000331033 }\end{array}$ & \begin{tabular}{|l|} 
Published online: \\
August 24, 2011
\end{tabular} & $\begin{array}{l}\text { @ } 2011 \text { S. Karger AG, Basel } \\
\text { ISSN 1662-0631 } \\
\text { www.karger.com/crg }\end{array}$ \\
\hline
\end{tabular}
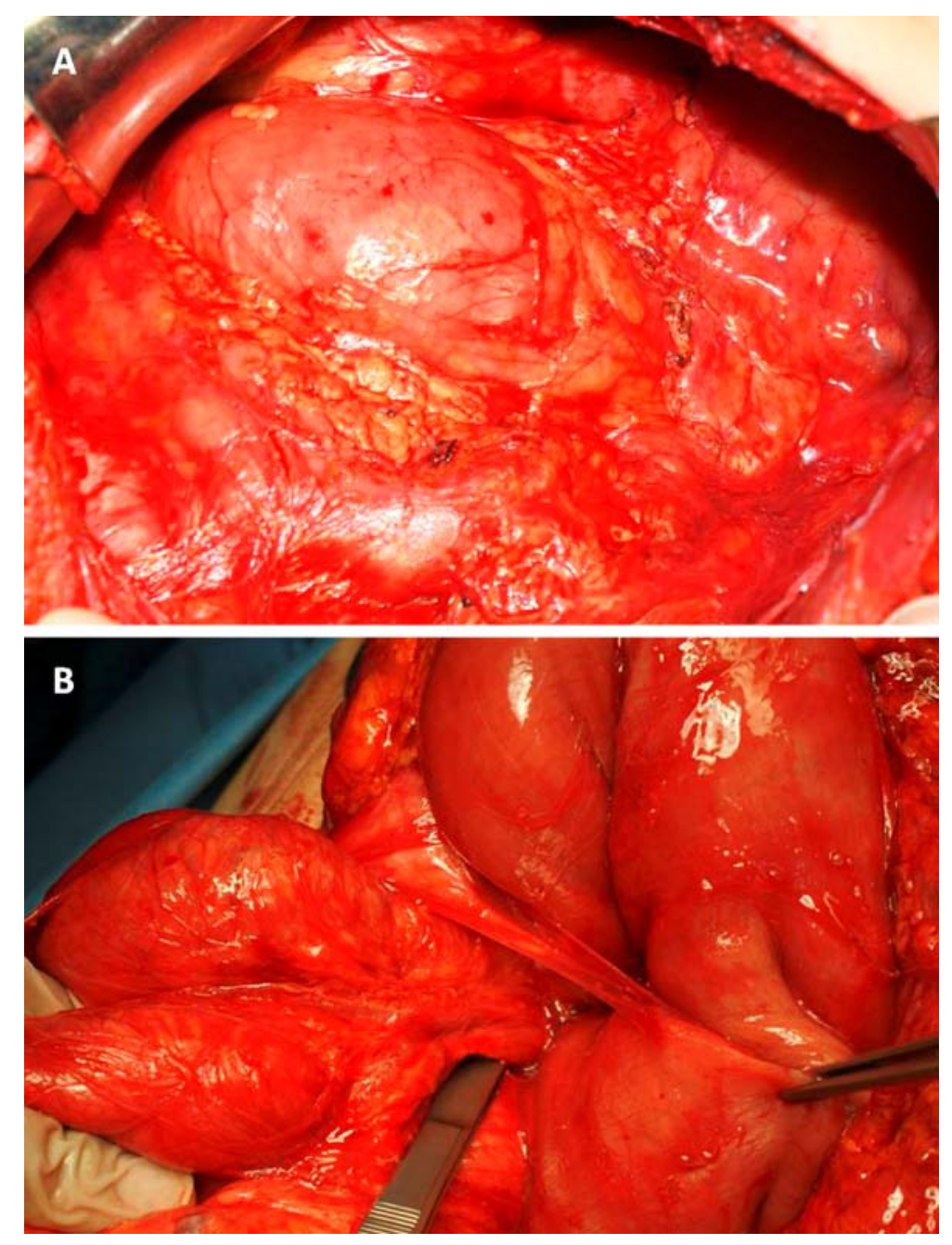

Fig. 3. Surgical findings. A At abdominal inspection, a sac containing dilated small bowel loops lateral to the duodenum, displacing the colon inferiorly, was seen. B Dilated small bowel loops protruding through the fossa of Waldeyer. The superior mesenteric vessels are identified anteriorly.

\section{References}

1 Stern LE, Warner BW: Congenital internal abdominal hernias: incidence and management; in Fitzgibbons RJ, Greenburg AG (eds): Nyhus and Condon's Hernia, 5th ed. Philadelphia, Lippincott Williams and Wilkins, 2002, pp 453-465.

- Ghahremani GG: Internal abdominal hernias. Surg Clin North Am 1984;64:393-406.

3 Hansmann GH, Morton SA: Intra-abdominal hernia: report of a case and review of the literature. Arch Surg 1939;39:973-986.

4 Bartlett MK, Wang C, Williams WH: The surgical management of paraduodenal hernia. Ann Surg 1968;168: 249-254.

5 Khan MA, Lo AY, Vande Maele DM: Paraduodenal hernia. Am Surg 1998;64:1218-1222.

6 Kurachi K, Nakamura T, Hayashi T, Asai Y, Kashiwabara T, Nakajima A, Suzuki S, Konno H: Left paraduodenal hernia in an adult complicated by ascending colon cancer: a case report. World J Gastroenterol 2006;12:1795-1797.

7 Cogswell HD, Thomas CA: Right paraduodenal hernia. Ann Surg 1941;114:1035-1041. 
8 Murray HOL: Left paraduodenal hernia. Can Med Assoc J 1955;72:263-267.

-9 Desjardins AU: Left paraduodenal hernia. Ann Surg 1918;67:195-201.

10 Zimmerman LM, Laufman H: Intraabdominal hernias due to developmental and rotational anomalies. Ann Surg 1953;138:82-91.

11 Taylor J: The X-ray diagnosis of right paraduodenal hernia. Br J Surg 1930;17:639-640.

12 Martin LC, Merkle EM, Thompson WM: Review of internal hernias: radiographic and clinical findings. AJR Am J Roentgenol 2006;186:703-717.

13 Shoji T, Nishiyama R, Oba K, Azuma M: Left paraduodenal hernia successfully treated with laparoscopic surgery: a case report. Case Rep Gastroenterol 2007;1:71-76.

14 McDonagh T, Jelinek GA: Two cases of paraduodenal hernia, a rare internal hernia. J Accid Emerg Med 1996;13:64-68.

15 Tong RSK, Sengupta S, Tjandra JJ: Left paraduodenal hernia: a case report and review of the literature. ANZ J Surg 2002;72:69-71. 